\title{
SAMPLE-PATH ANALYSIS OF THE PROPORTIONAL RELATION AND ITS CONSTANT FOR DISCRETE-TIME SINGLE-SERVER QUEUES
}

\author{
FUMIO ISHIZAKI AND NAOTO MIYOSHI
}

Received 22 December 2004; Revised 8 April 2005; Accepted 12 April 2005

In the previous work, the authors have considered a discrete-time queueing system and they have established that, under some assumptions, the stationary queue length distribution for the system with capacity $K_{1}$ is completely expressed in terms of the stationary distribution for the system with capacity $K_{0}\left(>K_{1}\right)$. In this paper, we study a sample-path version of this problem in more general setting, where neither stationarity nor ergodicity is assumed. We establish that, under some assumptions, the empirical queue length distribution (along through a sample path) for the system with capacity $K_{1}$ is completely expressed only in terms of the quantities concerning the corresponding system with capacity $K_{0}\left(>K_{1}\right)$. Further, we consider a probabilistic setting where the assumptions are satisfied with probability one, and under the probabilistic setting, we obtain a stochastic version of our main result. The stochastic version is considered as a generalization of the author's previous result, because the probabilistic assumptions are less restrictive.

Copyright (c) 2006 F. Ishizaki and N. Miyoshi. This is an open access article distributed under the Creative Commons Attribution License, which permits unrestricted use, distribution, and reproduction in any medium, provided the original work is properly cited.

\section{Introduction}

It is well known that a simple relation holds between the stationary queue length distribution of an $M / G I / 1 / K_{0}$ queue and that of the corresponding $M / G I / 1 / K_{1}$ queue (the same system except for the capacity $K_{1}<K_{0}$ ), that is,

$$
\mathrm{P}\left(X_{0}^{\left(K_{1}\right)}=j\right)=c \mathrm{P}\left(X_{0}^{\left(K_{0}\right)}=j\right) \quad \text { for } j=0, \ldots, K_{1}-1 \text {, }
$$

where $X_{0}^{\left(K_{i}\right)}, i=0,1$, denotes a random variable representing the steady-state queue length in the $M / G I / 1 / K_{i}$ queue and $c$ is a constant which is called proportional constant. We call such a relation the proportional relation. It has been shown that similar proportional relations also hold for some other queueing systems (see, e.g., $[1,2,6,7]$ ). 
In [7], the authors have considered a discrete-time stationary single-server queueing system described by the recursion;

$$
X_{n+1}=\min \left[\left(X_{n}-\delta_{n}\right)^{+}+A_{n+1}+B_{n+1}, K\right], \quad n \in \mathbb{Z},
$$

where $x^{+}=\max (x, 0)$. Here, $X_{n}(\in\{0, \ldots, K\})$ denotes the queue length at time $n$ and $\delta_{n}$ $(\in\{0,1\})$ represents the virtual departure, that is, a customer leaves the system at time $n$ only when $\delta_{n}=1$ and $X_{n}>0$, while no customer leaves at time $n$ when $\delta_{n}=0$. Both $A_{n}$ and $B_{n}$ denote the numbers of customers arriving at time $n$; the former is from a stationary arrival process with some regenerative structure and the latter is from an arrival process controlled according to the queue length. Under some assumptions for $\left\{A_{n}\right\}_{n \in \mathbb{Z}}$ and $\left\{B_{n}\right\}_{n \in \mathbb{Z}}$, the authors have not only established the proportional relations like (1.1) but also have shown that the proportional constant can be expressed in terms of the distribution for the system with capacity $K=K_{0}\left(>K_{1}\right)$. This implies that the stationary queue length distribution of a system with capacity $K_{1}$ can be completely expressed in terms of the distribution of the other system with capacity $K_{0}$ (see [7] for detail).

The proportional relation has rich applications when the proportional constant is completely expressed in terms of the distribution for one system. For instance, the proportional relation is useful for numerical method to compute performance measures in a finite-buffer queue (see, e.g., $[5,6]$ ) and for online concurrent performance estimation for queueing systems with different buffer capacities (see, e.g., $[3,4]$ ). In numerical computation, we can use the proportional relation to evaluate some performance measures of a finite-buffer queue in terms of the queue-length distribution of the corresponding infinite-buffer queue, where we exploit the fact that the distributions of infinite-buffer queues are, in general, computed in efficient and stable ways. On the other hand, in concurrent estimation, we can use the proportional relation to estimate the performance of multiple queueing systems with different buffer capacities simultaneously by simulating only one system.

In this paper, we study a sample-path (deterministic) version of the proportional relation in more general setting, where any probabilistic assumption (such as stationarity or ergodicity) is not needed. This is quite useful in applying our result to some online control of queueing systems such as dynamic buffer allocation. Our main result (Theorem 3.7) shows that under some assumptions (Assumptions 3.1 and 3.5), the empirical queue length distribution (along through a sample path) for the system with capacity $K_{1}$ is completely expressed only in terms of the quantities concerning the corresponding system with capacity $K_{0}\left(>K_{1}\right)$. In addition to the sample-path (deterministic) version, we consider a probabilistic setting where Assumptions 3.1 and 3.5 are satisfied with probability one. Under the probabilistic setting, we obtain a stochastic version (Corollary 4.2) of our main result. The stochastic version is considered as a generalization of [7, Theorem 1], because the probabilistic assumptions are less restrictive than those in [7].

The remainder of this paper is organized as follows. Section 2 describes a discretetime single-server queueing model considered in this paper. In Section 3, we first make a few assumptions and then show that under the assumptions, the empirical queue length 
distribution for the system with capacity $K_{1}$ is completely expressed only in terms of the quantities concerning the corresponding system with capacity $K_{0}\left(>K_{1}\right)$. In Section 4, we consider a probabilistic setting which satisfies the assumptions made in Section 3 and generalize [7, Theorem 1].

\section{Model}

Consider a discrete-time queueing system consisting of a single server and a buffer, where the buffer capacity may be finite or infinite and $K$ denotes the total system capacity (the buffer capacity plus one for the server). Time is divided into unit-time intervals called slots. Let $\left\{\gamma_{n}\right\}_{n \geq 1}$ denote an arrival process on $\mathbb{Z}_{+}$, where $\gamma_{n}$ represents the batch size (the number of customers) arriving at the beginning of the $n$th slot. An arriving customer that finds the buffer being full is discarded and lost. Let $\left\{\delta_{n}\right\}_{n \in \mathbb{Z}_{+}}$denote a sequence on $\{0,1\}$ closely related to the departure process, that is, $\delta_{n}$ represents the number of customers departing at the end of the $n$th slot when the system is not empty in the slot. Let $X_{n}(\in$ $\{0, \ldots, K\}), n \in \mathbb{Z}_{+}$, denote the queue length (including one in service if any) immediately after arrival in the $n$th slot. The dynamics of $\left\{X_{n}\right\}_{n \in \mathbb{Z}_{+}}$is then represented by the following recursion with an initial state $X_{0}$;

$$
X_{n+1}=\min \left[\left(X_{n}-\delta_{n}\right)^{+}+\gamma_{n+1}, K\right], \quad n \in \mathbb{Z}_{+} .
$$

We can interpret $\left\{\left(X_{n}, \delta_{n}, \gamma_{n+1}\right)\right\}_{n \in \mathbb{Z}_{+}}$as a sample path extracted from the corresponding stochastic process, where neither stationarity nor ergodicity is assumed.

Hereafter, we consider two queueing systems both of which are identical to the above but their system capacities are $K_{0}$ and $K_{1}$, respectively, where $0<K_{1}<K_{0} \leq+\infty$. We refer to the system with capacity $K_{i}$ as $K_{i}$-system for $i=0,1$, and to emphasize the system capacity, we put the superscript $\left(K_{i}\right)$ on the quantities associated with the $K_{i}$-system.

\section{Main result}

For any event sequence $\left\{E_{n}\right\}_{n \in \mathbb{Z}_{+}}$, let $\mathscr{P}\left(E_{n}\right)$ denote the long-run average of the indicator $1_{E_{n}}$, that is,

$$
\mathscr{P}\left(E_{n}\right)=\lim _{m \rightarrow \infty} \frac{1}{m} \sum_{n=0}^{m-1} 1_{E_{n}},
$$

provided that the limit exists. Note that the subscript $n$ has no rigorous meaning in the left-hand side of (3.1), which is just the abbreviation of $\mathscr{P}\left(\left\{E_{n}\right\}_{n \in \mathbb{Z}_{+}}\right)$. In this section, we show under some mild assumptions that $\mathscr{P}\left(X_{n}^{\left(K_{1}\right)}=j\right), j=0, \ldots, K_{1}$, is (if it exists) completely expressed in terms of the long-run averages concerning the $K_{0}$-system. The proof consists of two steps: first, we show in Section 3.1 that $\mathscr{P}\left(X_{n}^{\left(K_{0}\right)}=j\right)$ and $\mathscr{P}\left(X_{n}^{\left(K_{1}\right)}=\right.$ $j), j=0, \ldots, K_{1}-1$, are proportionally related. This part may be straightforward from the classical truncation property of censored processes. However, this is not yet enough to say that $\mathscr{P}\left(X_{n}^{\left(K_{1}\right)}=j\right), j=0, \ldots, K_{1}$, is completely expressed in terms of the long-run averages concerning the $K_{0}$-system. Then in Section 3.2, we verify that the proportional constant is also expressed in terms of the long-run averages concerning the $K_{0}$-system. 
3.1. Proportional relation. First we put a basic assumption.

Assumption 3.1. (i) For $j=0,1, \ldots, K_{1}$ and $i=0,1, \mathscr{P}\left(X_{n}^{\left(K_{i}\right)}=j\right)$ exists. In addition, for at least one $j=0,1, \ldots, K_{1}-1$, both $\mathscr{P}\left(X_{n}^{\left(K_{0}\right)}=j\right)$ and $\mathscr{P}\left(X_{n}^{\left(K_{1}\right)}=j\right)$ are positive,

(ii) there exist $N_{0}$ and $N_{1} \in \mathbb{Z}_{+}$such that $X_{N_{0}}^{\left(K_{0}\right)}=X_{N_{1}}^{\left(K_{1}\right)}$.

The latter part of Assumption 3.1(i) excludes the cases where $\mathscr{P}\left(X_{n}^{\left(K_{1}\right)}=K_{1}\right)=1$ or $\mathscr{P}\left(X_{n}^{\left(K_{0}\right)} \geq K_{1}\right)=1$. Assumption 3.1(ii) ensures that the set of possible states of $\left\{X_{n}^{\left(K_{0}\right)}\right\}_{n \in \mathbb{Z}_{+}}$ and that of $\left\{X_{n}^{\left(K_{1}\right)}\right\}_{n \in \mathbb{Z}_{+}}$are not disjoint.

We define a sequence $\left\{\phi\left(m, K_{i}\right)\right\}_{m \geq 1}, i=0,1$, by

$$
\phi\left(m, K_{i}\right)=\min \left\{l \geq N_{i}: \sum_{n=N_{i}}^{l} 1_{\left\{X_{n}^{\left(K_{i}\right)} \leq K_{1}-1\right\}}=m\right\} .
$$

In other words, $\phi\left(m, K_{i}\right)$ represents the first epoch at which the cumulative time of $\left\{X_{n}^{\left(K_{i}\right)} \leq\right.$ $K_{1}-1$ \} reaches $m$ after (and including) $N_{i}$. Note that under Assumption 3.1, $\phi\left(m, K_{i}\right)$ exists for every $m=1,2, \ldots$ and $i=0,1$, and $\lim _{m \rightarrow \infty} \phi\left(m, K_{i}\right)=+\infty$.

The next assumption, which imposes a restriction on the arrival and the departure processes, is a key to establish the following lemma.

Assumption 3.2. (i) For all $m \geq 1, \delta_{\phi\left(m, K_{0}\right)}^{\left(K_{0}\right)}=\delta_{\phi\left(m, K_{1}\right)}^{\left(K_{1}\right)}$ whenever $X_{\phi\left(m, K_{0}\right)}^{\left(K_{0}\right)}>0$,

(ii) for all $m \geq 1, \gamma_{\phi\left(m, K_{0}\right)+1}^{\left(K_{0}\right)}=\gamma_{\phi\left(m, K_{1}\right)+1}^{\left(K_{1}\right)}$.

In [7], it is assumed in stationary setting that $\left\{\left(\delta_{n}^{\left(K_{0}\right)}, A_{n+1}^{\left(K_{0}\right)}\right)\right\}_{n \in \mathbb{Z}}$ and $\left\{\left(\delta_{n}^{\left(K_{1}\right)}, A_{n+1}^{\left(K_{1}\right)}\right)\right\}_{n \in \mathbb{Z}}$ are stochastically identical, where $\delta_{n}^{\left(K_{i}\right)}$ and $A_{n+1}^{\left(K_{i}\right)}, i=0,1$, are $\delta_{n}$ and $A_{n+1}$ in (1.2) for $K=K_{i}$, respectively. However, we here impose the corresponding consistency only for $n \geq N_{i}$ such that $X_{n}^{\left(K_{i}\right)} \leq K_{1}-1$.

Lemma 3.3. Under Assumptions 3.1 and 3.2, for all $m \geq 1$,

$$
X_{\phi\left(m, K_{0}\right)}^{\left(K_{0}\right)}=X_{\phi\left(m, K_{1}\right)}^{\left(K_{1}\right)}
$$

Proof. We prove Lemma 3.3 by induction. Consider the case of $m=1$. If $X_{N_{i}}^{\left(K_{i}\right)} \leq K_{1}-1$ for $i=0,1$ under Assumption 3.1, then $\phi\left(1, K_{i}\right)=N_{i}$ by the definition (3.2) and we see that $X_{\phi\left(1, K_{0}\right)}^{\left(K_{0}\right)}=X_{\phi\left(1, K_{1}\right)}^{\left(K_{1}\right)}$. On the other hand, if $X_{N_{i}}^{\left(K_{i}\right)}=K_{1}$, then due to the property of "skip free to the left" for $\left\{X_{n}^{\left(K_{i}\right)}\right\}_{n \in \mathbb{Z}_{+}}$, we have $X_{\phi\left(1, K_{0}\right)}^{\left(K_{0}\right)}=X_{\phi\left(1, K_{1}\right)}^{\left(K_{1}\right)}=K_{1}-1$, where the existence of a finite $\phi\left(1, K_{i}\right), i=0,1$, is ensured by Assumption 3.1.

We now suppose that (3.3) holds for some $m \geq 1$. If $\left(X_{\phi\left(m, K_{0}\right)}^{\left(K_{0}\right)}-\delta_{\phi\left(m, K_{0}\right)}^{\left(K_{0}\right)}\right)^{+}+\gamma_{\phi\left(m, K_{0}\right)+1}^{\left(K_{0}\right)} \leq$ $K_{1}-1$, then due to the system dynamics (2.1), we have under Assumption 3.2 that

$$
X_{\phi\left(m, K_{0}\right)+1}^{\left(K_{0}\right)}=X_{\phi\left(m, K_{1}\right)+1}^{\left(K_{1}\right)} \leq K_{1}-1
$$

We thus see that $\phi\left(m+1, K_{i}\right)=\phi\left(m, K_{i}\right)+1$ for $i=0,1$, and conclude that $X_{\phi\left(m+1, K_{0}\right)}^{\left(K_{0}\right)}=$ $X_{\phi\left(m+1, K_{1}\right)}^{\left(K_{1}\right)}$. On the other hand, if $\left(X_{\phi\left(m, K_{0}\right)}^{\left(K_{0}\right)}-\delta_{\phi\left(m, K_{0}\right)}^{\left(K_{0}\right)}\right)^{+}+\gamma_{\phi\left(m, K_{0}\right)+1}^{\left(K_{0}\right)} \geq K_{1}$, then due to the 
property of "skip free to the left" for $\left\{X_{n}^{\left(K_{i}\right)}\right\}_{n \in \mathbb{Z}_{+}}, i=0,1$, we conclude that

$$
X_{\phi\left(m+1, K_{0}\right)}^{\left(K_{0}\right)}=X_{\phi\left(m+1, K_{1}\right)}^{\left(K_{1}\right)}=K_{1}-1
$$

where the existence of a finite $\phi\left(m+1, K_{i}\right), i=0,1$, is ensured by Assumption 3.1.

Lemma 3.3 shows that the censored (truncated) process of $\left\{X_{n}^{\left(K_{0}\right)}\right\}_{n \in \mathbb{Z}_{+}}$such that the state space is truncated at $K_{1}-1$ coincides with that of $\left\{X_{n}^{\left(K_{1}\right)}\right\}_{n \in \mathbb{Z}_{+}}$under the stated assumptions. Lemma 3.3 readily leads to the goal of this section.

Lemma 3.4. Under Assumptions 3.1 and 3.2, for $j=0, \ldots, K_{1}-1$,

$$
C_{0} \mathscr{P}\left(X_{n}^{\left(K_{0}\right)}=j\right)=C_{1} \mathscr{P}\left(X_{n}^{\left(K_{1}\right)}=j\right)
$$

where $C_{i}, i=0,1$, denotes the limit

$$
C_{i}=\lim _{m \rightarrow \infty} \frac{\phi\left(m, K_{i}\right)}{m}
$$

Proof. First, we claim the existence of $C_{i}, i=0,1$, in (3.7). Note that, under Assumption 3.1, $\mathscr{P}\left(X_{n}^{\left(K_{i}\right)} \leq K_{1}-1\right)$ exists and is positive, and $\lim _{m \rightarrow \infty} \phi\left(m, K_{i}\right)=+\infty$ for $i=0,1$. Then, using $\sum_{n=N_{i}}^{\phi\left(m, K_{i}\right)} 1_{\left\{X_{n}^{\left(K_{i}\right)} \leq K_{1}-1\right\}}=m$ for $i=0,1$, by the definition of $\phi$, we obtain

$$
\begin{aligned}
\mathscr{P}\left(X_{n}^{\left(K_{i}\right)} \leq K_{1}-1\right) & =\lim _{m \rightarrow \infty} \frac{1}{\phi\left(m, K_{i}\right)}\left[\sum_{n=N_{i}}^{\phi\left(m, K_{i}\right)} 1_{\left\{X_{n}^{\left(K_{i}\right)} \leq K_{1}-1\right\}}+\sum_{n=1}^{N_{i}-1} 1_{\left\{X_{n}^{\left(K_{i}\right)} \leq K_{1}-1\right\}}\right] \\
& =\lim _{m \rightarrow \infty} \frac{m}{\phi\left(m, K_{i}\right)}=\frac{1}{C_{i}} .
\end{aligned}
$$

We therefore see that $C_{i}$ exists and is equal to $1 / P\left(X_{n}^{\left(K_{i}\right)} \leq K_{1}-1\right)(\geq 1)$ for $i=0,1$.

Now, we show the relation (3.6). Similar to the above, we have for $i=0,1$, and $j=$ $0, \ldots, K_{1}-1$,

$$
\mathscr{P}\left(X_{n}^{\left(K_{i}\right)}=j\right)=\lim _{m \rightarrow \infty} \frac{1}{\phi\left(m, K_{i}\right)} \sum_{n=N_{i}}^{\phi\left(m, K_{i}\right)} 1_{\left\{X_{n}^{\left(K_{i}\right)}=j\right\}}
$$

Here, from Lemma 3.3, we have $\sum_{n=N_{0}}^{\phi\left(m, K_{0}\right)} 1_{\left\{X_{n}^{\left(K_{0}\right)}=j\right\}}=\sum_{n=N_{1}}^{\phi\left(m, K_{1}\right)} 1_{\left\{X_{n}^{\left(K_{1}\right)}=j\right\}}$ for $m \geq 1$ and $j=$ $0, \ldots, K_{1}-1$, and thus, dividing both the sides by $m$,

$$
\frac{\phi\left(m, K_{0}\right)}{m} \frac{1}{\phi\left(m, K_{0}\right)} \sum_{n=N_{0}}^{\phi\left(m, K_{0}\right)} 1_{\left\{X_{n}^{\left(K_{0}\right)}=j\right\}}=\frac{\phi\left(m, K_{1}\right)}{m} \frac{1}{\phi\left(m, K_{1}\right)} \sum_{n=N_{1}}^{\phi\left(m, K_{1}\right)} 1_{\left\{X_{n}^{\left(K_{1}\right)}=j\right\}} .
$$

Hence, taking $m$ to infinity, we derive (3.6). 
3.2. Proportional constant. In general, to determine the proportional constant $C_{0} / C_{1}$ appearing in (3.6), we need some quantities for both $K_{0^{-}}$and $K_{1}$-systems, for example, both $\mathscr{P}\left(X_{n}^{\left(K_{0}\right)} \leq K_{1}-1\right)$ and $\mathscr{P}\left(X_{n}^{\left(K_{1}\right)} \leq K_{1}-1\right)$. However, with some additional assumptions, we can express the proportional constant $C_{0} / C_{1}$ only in terms of the quantities concerning $K_{0}$-system. For such a purpose, we replace Assumption 3.2 with the following.

Assumption 3.5. (i) For all $m \geq 1, \delta_{\phi\left(m, K_{0}\right)}^{\left(K_{0}\right)}=\delta_{\phi\left(m, K_{1}\right)}^{\left(K_{1}\right)}$,

(ii) the arrival process $\left\{\gamma_{n}^{\left(K_{i}\right)}\right\}_{n \geq 1}, i=0,1$, is separable such that $\gamma_{n}^{\left(K_{i}\right)}=A_{n}^{\left(K_{i}\right)}+B_{n}^{\left(K_{i}\right)}$, $A_{n}^{\left(K_{i}\right)} \geq 0, B_{n}^{\left(K_{i}\right)} \geq 0, n \geq 1$, where

$$
\left(A_{\phi\left(m, K_{0}\right)+1}^{\left(K_{0}\right)}, B_{\phi\left(m, K_{0}\right)+1}^{\left(K_{0}\right)}\right)=\left(A_{\phi\left(m, K_{1}\right)+1}^{\left(K_{1}\right)}, B_{\phi\left(m, K_{1}\right)+1}^{\left(K_{1}\right)}\right) .
$$

Furthermore, the following relations hold together with the existence of the limits:

$$
\begin{aligned}
\mathscr{P}\left(X_{n}^{\left(K_{i}\right)}=K_{1}\right) \mathscr{P}\left(X_{n}^{\left(K_{i}\right)}=K_{1}, \delta_{n}^{\left(K_{i}\right)}=1, \gamma_{n+1}^{\left(K_{i}\right)}=0\right) \\
\quad=\mathscr{P}\left(X_{n}^{\left(K_{i}\right)}=K_{1}, B_{n+1}^{\left(K_{i}\right)}=0\right) \mathscr{P}\left(X_{n}^{\left(K_{i}\right)}=K_{1}, \delta_{n}^{\left(K_{i}\right)}=1, A_{n+1}^{\left(K_{i}\right)}=0\right) .
\end{aligned}
$$

Also,

$$
\begin{gathered}
\mathscr{P}\left(\delta_{n}^{\left(K_{0}\right)}=1, A_{n+1}^{\left(K_{0}\right)}=0\right)=\mathscr{P}\left(\delta_{n}^{\left(K_{1}\right)}=1, A_{n+1}^{\left(K_{1}\right)}=0\right), \\
\frac{\mathscr{P}\left(X_{n}^{\left(K_{0}\right)}=K_{1}, B_{n+1}^{\left(K_{0}\right)}=0\right)}{\mathscr{P}\left(X_{n}^{\left(K_{0}\right)}=K_{1}\right)}=\frac{\mathscr{P}\left(X_{n}^{\left(K_{1}\right)}=K_{1}, B_{n+1}^{\left(K_{1}\right)}=0\right)}{\mathscr{P}\left(X_{n}^{\left(K_{1}\right)}=K_{1}\right)},
\end{gathered}
$$

provided that the denominators are positive.

Note that Assumption 3.5(i) slightly strengthens Assumption 3.2(i). Also, (3.11) corresponds to Assumption 3.2(ii) and is the consequence of separating $\left\{\gamma_{n}^{\left(K_{i}\right)}\right\}_{n \geq 1}$. Therefore, Lemmas 3.3 and 3.4 remain to hold under Assumptions 3.1 and 3.5. The intuitive meaning of (3.12) is that the empirical conditional probability of $B_{n+1}^{\left(K_{i}\right)}=0$, given $X_{n}^{\left(K_{i}\right)}=K_{1}$, is irrelevant to the event $\left\{\delta_{n}^{\left(K_{i}\right)}=1, A_{n+1}^{\left(K_{i}\right)}=0\right\}$ for $i=0,1$. Also, (3.13) and (3.14) mean that the empirical probabilities of those events are identical in $K_{0}$ - and $K_{1}$ systems.

The following lemma shows that the proportional constant $C_{0} / C_{1}$ can be expressed only in terms of the quantities concerning $K_{0}$-system under Assumptions 3.1 and 3.5.

Lemma 3.6. Under Assumptions 3.1 and 3.5, we have

$$
\frac{C_{0}}{C_{1}}= \begin{cases}\frac{\mathscr{P}\left(\delta_{n}^{\left(K_{0}\right)}=1, A_{n+1}^{\left(K_{0}\right)}=0\right)}{\mathscr{P}\left(X_{n}^{\left(K_{0}\right)} \leq K_{1}, \delta_{n}^{\left(K_{0}\right)}=1, A_{n+1}^{\left(K_{0}\right)}=0\right)} & \text { if } \mathscr{P}\left(\delta_{n}^{\left(K_{0}\right)}=1, A_{n+1}^{\left(K_{0}\right)}=0\right)>0, \\ 1, & \text { otherwise. }\end{cases}
$$

Proof. If $\mathscr{P}\left(\delta_{n}^{\left(K_{0}\right)}=1, A_{n+1}^{\left(K_{0}\right)}=0\right)=0$, then under Assumption 3.1, $\mathscr{P}\left(X_{n}^{\left(K_{0}\right)}=j^{*}\right)=\mathscr{P}\left(X_{n}^{\left(K_{1}\right)}=\right.$ $\left.j^{*}\right)=1$, and the result is trivial, where $j^{*}$ is such that $j^{*}=X_{N_{0}}^{\left(K_{0}\right)}=X_{N_{1}}^{\left(K_{1}\right)}$ in Assumption 3.1. 
Suppose that $\mathscr{P}\left(\delta_{n}^{\left(K_{0}\right)}=1, A_{n+1}^{\left(K_{0}\right)}=0\right)>0$. Then, we show below that

$$
\begin{gathered}
C_{0} \mathscr{P}\left(X_{n}^{\left(K_{0}\right)} \leq K_{1}-1, \delta_{n}^{\left(K_{0}\right)}=1, A_{n+1}^{\left(K_{0}\right)}=0\right)=C_{1} \mathscr{P}\left(X_{n}^{\left(K_{1}\right)} \leq K_{1}-1, \delta_{n}^{\left(K_{1}\right)}=1, A_{n+1}^{\left(K_{1}\right)}=0\right) \\
C_{0} \mathscr{P}\left(X_{n}^{\left(K_{0}\right)}=K_{1}, \delta_{n}^{\left(K_{0}\right)}=1, A_{n+1}^{\left(K_{0}\right)}=0\right)=C_{1} \mathscr{P}\left(X_{n}^{\left(K_{1}\right)}=K_{1}, \delta_{n}^{\left(K_{1}\right)}=1, A_{n+1}^{\left(K_{1}\right)}=0\right),
\end{gathered}
$$

together with the existence of the limits in (3.16) (note that the existence of the limits in (3.17) is assumed in (3.12)). Once (3.16) and (3.17) are provided, summing each side of them and noting that $\left\{X_{n}^{\left(K_{1}\right)} \leq K_{1}\right\}$ is certain, we obtain

$$
C_{0} \mathscr{P}\left(X_{n}^{\left(K_{0}\right)} \leq K_{1}, \delta_{n}^{\left(K_{0}\right)}=1, A_{n+1}^{\left(K_{0}\right)}=0\right)=C_{1} \mathscr{P}\left(\delta_{n}^{\left(K_{1}\right)}=1, A_{n+1}^{\left(K_{1}\right)}=0\right)
$$

Therefore, (3.15) is derived by applying (3.13).

We first derive (3.16). From Lemma 3.3 with Assumptions 3.1 and 3.5,

$$
\sum_{n=N_{0}}^{\phi\left(m, K_{0}\right)} 1_{\left\{X_{n}^{\left(K_{0}\right)} \leq K_{1}-1, \delta_{n}^{\left(K_{0}\right)}=1, A_{n+1}^{\left(K_{0}\right)}=0\right\}}=\sum_{n=N_{1}}^{\phi\left(m, K_{1}\right)} 1_{\left\{X_{n}^{\left(K_{1}\right)} \leq K_{1}-1, \delta_{n}^{\left(K_{1}\right)}=1, A_{n+1}^{\left(K_{1}\right)}=0\right\}}
$$

Thus, dividing both the sides by $m$ and taking $m$ to infinity, we have (3.16) under Assumptions 3.1 and 3.5, where the existence of the limits in (3.16) is ensured since

$$
\begin{aligned}
& \mathscr{P}\left(X_{n}^{\left(K_{1}\right)} \leq K_{1}-1, \delta_{n}^{\left(K_{1}\right)}=1, A_{n+1}^{\left(K_{1}\right)}=0\right) \\
& \quad=\mathscr{P}\left(\delta_{n}^{\left(K_{1}\right)}=1, A_{n+1}^{\left(K_{1}\right)}=0\right)-\mathscr{P}\left(X_{n}^{\left(K_{1}\right)}=K_{1}, \delta_{n}^{\left(K_{1}\right)}=1, A_{n+1}^{\left(K_{1}\right)}=0\right),
\end{aligned}
$$

and the two terms in the right-hand side above exist under Assumption 3.5.

Now, we show (3.17). From Lemma 3.3 with Assumptions 3.1 and 3.5, we have

$$
\sum_{n=N_{0}}^{\phi\left(m, K_{0}\right)} 1_{\left\{X_{n}^{\left(K_{0}\right)} \leq K_{1}-1, X_{n+1}^{\left(K_{0}\right)} \geq K_{1}\right\}}=\sum_{n=N_{1}}^{\phi\left(m, K_{1}\right)} 1_{\left\{X_{n}^{\left(K_{1}\right)} \leq K_{1}-1, X_{n+1}^{\left(K_{1}\right)} \geq K_{1}\right\}} .
$$

Thus, dividing both the sides by $m$ and taking $m$ to infinity, we have under Assumption 3.1 ,

$$
C_{0} \mathscr{P}\left(X_{n}^{\left(K_{0}\right)} \leq K_{1}-1, X_{n+1}^{\left(K_{0}\right)} \geq K_{1}\right)=C_{1} \mathscr{P}\left(X_{n}^{\left(K_{1}\right)} \leq K_{1}-1, X_{n+1}^{\left(K_{1}\right)} \geq K_{1}\right)
$$

provided that the limits exist. When the limits above exist, due to the level-crossing argument under Assumption 3.1, we have for $i=0,1$,

$$
\begin{aligned}
\mathscr{P}\left(X_{n}^{\left(K_{i}\right)} \leq K_{1}-1, X_{n+1}^{\left(K_{i}\right)} \geq K_{1}\right) & =\mathscr{P}\left(X_{n}^{\left(K_{i}\right)} \geq K_{1}, X_{n+1}^{\left(K_{i}\right)} \leq K_{1}-1\right) \\
& =\mathscr{P}\left(X_{n}^{\left(K_{i}\right)}=K_{1}, \delta_{n}^{\left(K_{i}\right)}=1, \gamma_{n+1}^{\left(K_{i}\right)}=0\right),
\end{aligned}
$$


where the second equality follows from (2.1) through the property of "skip free to the left" for $\left\{X_{n}^{\left(K_{i}\right)}\right\}_{n \in \mathbb{Z}_{+}}, i=0,1$. We here see that all the limits in (3.23) (and then those in (3.22)) exist because $\mathscr{P}\left(X_{n}^{\left(K_{i}\right)}=K_{1}, \delta_{n}^{\left(K_{i}\right)}=1, \gamma_{n+1}^{\left(K_{i}\right)}=0\right), i=0,1$, is assumed to exist in Assumption 3.5. From (3.22) and (3.23), we have

$$
C_{0} \mathscr{P}\left(X_{n}^{\left(K_{0}\right)}=K_{1}, \delta_{n}^{\left(K_{0}\right)}=1, \gamma_{n+1}^{\left(K_{0}\right)}=0\right)=C_{1} \mathscr{P}\left(X_{n}^{\left(K_{1}\right)}=K_{1}, \delta_{n}^{\left(K_{1}\right)}=1, \gamma_{n+1}^{\left(K_{1}\right)}=0\right)
$$

Hereafter, we separately treat the three cases: (a) $\mathscr{P}\left(X_{n}^{\left(K_{0}\right)}=K_{1}\right)>0$ and $\mathscr{P}\left(X_{n}^{\left(K_{1}\right)}=\right.$ $\left.K_{1}\right)>0$, (b) either $\mathscr{P}\left(X_{n}^{\left(K_{0}\right)}=K_{1}\right)=0$ or $\mathscr{P}\left(X_{n}^{\left(K_{1}\right)}=K_{1}\right)=0$, and (c) $\mathscr{P}\left(X_{n}^{\left(K_{0}\right)}=K_{1}\right)=$ $\mathscr{P}\left(X_{n}^{\left(K_{1}\right)}=K_{1}\right)=0$. In the case (a), we have from (3.12) that, for $i=0,1$,

$$
\begin{aligned}
\mathscr{P}\left(X_{n}^{\left(K_{i}\right)}=K_{1}, \delta_{n}^{\left(K_{i}\right)}=1, \gamma_{n+1}^{\left(K_{i}\right)}=0\right) \\
\quad=\mathscr{P}\left(X_{n}^{\left(K_{i}\right)}=K_{1}, \delta_{n}^{\left(K_{i}\right)}=1, A_{n+1}^{\left(K_{i}\right)}=0\right) \frac{\mathscr{P}\left(X_{n}^{\left(K_{i}\right)}=K_{1}, B_{n+1}^{\left(K_{i}\right)}=0\right)}{\mathscr{P}\left(X_{n}^{\left(K_{i}\right)}=K_{1}\right)} .
\end{aligned}
$$

Hence, substituting (3.25) into (3.24) and then using (3.14), we derive (3.17).

Next we consider the case (b). Suppose here that $\mathscr{P}\left(X_{n}^{\left(K_{0}\right)}=K_{1}\right)>0$ and $\mathscr{P}\left(X_{n}^{\left(K_{1}\right)}=\right.$ $\left.K_{1}\right)=0$. Since $\mathscr{P}\left(X_{n}^{\left(K_{1}\right)}=K_{1}, \delta_{n}^{\left(K_{1}\right)}=1, \gamma_{n+1}^{\left(K_{1}\right)}=0\right)=0$ in this case, using (3.12) only in the left-hand side of (3.24), we obtain

$$
C_{0} \mathscr{P}\left(X_{n}^{\left(K_{0}\right)}=K_{1}, \delta_{n}^{\left(K_{0}\right)}=1, A_{n+1}^{\left(K_{0}\right)}=0\right) \frac{\mathscr{P}\left(X_{n}^{\left(K_{0}\right)}=K_{1}, B_{n+1}^{\left(K_{0}\right)}=0\right)}{\mathscr{P}\left(X_{n}^{\left(K_{0}\right)}=K_{1}\right)}=0 .
$$

Here, if $\mathscr{P}\left(X_{n}^{\left(K_{0}\right)}=K_{1}\right)>0$ and $\mathscr{P}\left(X_{n}^{\left(K_{0}\right)}=K_{1}, B_{n+1}^{\left(K_{0}\right)}=0\right)=0$, it contradicts with $\mathscr{P}\left(X_{n}^{\left(K_{0}\right)} \leq\right.$ $\left.K_{1}-1\right)>0$ under Assumption 3.1. Thus, we have $\mathscr{P}\left(X_{n}^{\left(K_{0}\right)}=K_{1}, B_{n+1}^{\left(K_{0}\right)}=0\right)>0$. Also, recalling that $C_{0}>0$ under Assumption 3.1, we see from (3.26) that

$$
\mathscr{P}\left(X_{n}^{\left(K_{0}\right)}=K_{1}, \delta_{n}^{\left(K_{0}\right)}=1, A_{n+1}^{\left(K_{0}\right)}=0\right)=0 .
$$

On the other hand, $\mathscr{P}\left(X_{n}^{\left(K_{1}\right)}=K_{1}\right)=0$ clearly implies

$$
\mathscr{P}\left(X_{n}^{\left(K_{1}\right)}=K_{1}, \delta_{n}^{\left(K_{1}\right)}=1, A_{n+1}^{\left(K_{1}\right)}=0\right)=0
$$

and (3.17) follows. For the case of $\mathscr{P}\left(X_{n}^{\left(K_{0}\right)}=K_{1}\right)=0$ and $\mathscr{P}\left(X_{n}^{\left(K_{1}\right)}=K_{1}\right)>0$, we can also derive (3.17) by the similar argument.

Finally, in the case $(\mathrm{c}), \mathscr{P}\left(X_{n}^{\left(K_{i}\right)}=K_{1}, \delta_{n}^{\left(K_{i}\right)}=1, A_{n+1}^{\left(K_{i}\right)}=0\right)=0$ for $i=0,1$, and (3.17) immediately follows. 
The following theorem is a direct conclusion of Lemmas 3.4 and 3.6.

Theorem 3.7. Suppose that Assumptions 3.1 and 3.5 hold. If $\mathscr{P}\left(\delta_{n}^{\left(K_{0}\right)}=1, A_{n+1}^{\left(K_{0}\right)}=0\right)=0$, then there exists some $j^{*} \in\left\{0, \ldots, K_{1}-1\right\}$ such that $\mathscr{P}\left(X_{n}^{\left(K_{0}\right)}=j^{*}\right)=\mathscr{P}\left(X_{n}^{\left(K_{1}\right)}=j^{*}\right)=1$. On the other hand, if $\mathscr{P}\left(\delta_{n}^{\left(K_{0}\right)}=1, A_{n+1}^{\left(K_{0}\right)}=0\right)>0$, then

$$
\begin{gathered}
\mathscr{P}\left(X_{n}^{\left(K_{1}\right)}=j\right)=\frac{\mathscr{P}\left(\delta_{n}^{\left(K_{0}\right)}=1, A_{n+1}^{\left(K_{0}\right)}=0\right) \mathscr{P}\left(X_{n}^{\left(K_{0}\right)}=j\right)}{\mathscr{P}\left(X_{n}^{\left(K_{0}\right)} \leq K_{1}, \delta_{n}^{\left(K_{0}\right)}=1, A_{n+1}^{\left(K_{0}\right)}=0\right)}, \quad \text { for } j=0, \ldots, K_{1}-1, \\
\mathscr{P}\left(X_{n}^{\left(K_{1}\right)}=K_{1}\right)=1-\frac{\mathscr{P}\left(\delta_{n}^{\left(K_{0}\right)}=1, A_{n+1}^{\left(K_{0}\right)}=0\right) \mathscr{P}\left(X_{n}^{\left(K_{0}\right)} \leq K_{1}-1\right)}{\mathscr{P}\left(X_{n}^{\left(K_{0}\right)} \leq K_{1}, \delta_{n}^{\left(K_{0}\right)}=1, A_{n+1}^{\left(K_{0}\right)}=0\right)} .
\end{gathered}
$$

\section{Stochastic version of our main result}

In Section 3, we have established Theorem 3.7, which may be considered as a sample-path version of [7, Theorem 1]. From this point of view, Assumption 3.1 in this paper corresponds to [7, Assumption 2(ii)], and Assumption 3.5 in this paper corresponds to [7, Assumptions 1(ii) and 2(i)]. However, we can consider probabilistic settings that satisfy Assumption 3.5 with probability one and are less restrictive than [7, Assumptions 1(ii) and 2(i)]. The following assumption is an example of such probabilistic settings.

Assumption 4.1. (i) For $n \in \mathbb{Z}_{+}$, given $X_{n}^{\left(K_{i}\right)}, i=0,1, B_{n+1}^{\left(K_{i}\right)}$ is conditionally independent of all other random elements. Further, $\mathrm{P}\left(B_{\phi\left(n, K_{0}\right)+1}^{\left(K_{0}\right)}=k \mid X_{\phi\left(n, K_{0}\right)}^{\left(K_{0}\right)}=j\right)=\mathrm{P}\left(B_{\phi\left(n, K_{1}\right)+1}^{\left(K_{1}\right)}=k \mid\right.$ $\left.X_{\phi\left(n, K_{1}\right)}^{\left(K_{1}\right)}=j\right)$ for all $j \in\left\{0, \ldots, K_{1}-1\right\}, k \in \mathbb{Z}_{+}$and $n \in \mathbb{Z}$. In addition, $\mathrm{P}\left(B_{n+1}^{\left(K_{i}\right)}=0 \mid X_{n}^{\left(K_{i}\right)}=\right.$ $\left.K_{1}\right), i=0,1$, is invariant in $n \in \mathbb{Z}_{+}$and $\mathrm{P}\left(B_{n+1}^{\left(K_{0}\right)}=0 \mid X_{n}^{\left(K_{0}\right)}=K_{1}\right)=\mathrm{P}\left(B_{n+1}^{\left(K_{1}\right)}=0 \mid X_{n}^{\left(K_{1}\right)}=K_{1}\right)$,

(ii) for all $n \in \mathbb{Z}, i \in\{0,1\}$ and $j \in \mathbb{Z}_{+}, \mathrm{P}\left(\delta_{\phi\left(n, K_{0}\right)}^{\left(K_{0}\right)}=i, A_{\phi\left(n, K_{0}\right)+1}^{\left(K_{0}\right)}=j\right)=\mathrm{P}\left(\delta_{\phi\left(n, K_{1}\right)}^{\left(K_{1}\right)}=\right.$ $\left.i, A_{\phi\left(n, K_{1}\right)+1}^{\left(K_{1}\right)}=j\right)$. In addition, for all $n \in \mathbb{Z}_{+}, \mathrm{P}\left(\delta_{n}^{\left(K_{0}\right)}=1, A_{n+1}^{\left(K_{0}\right)}=0\right)=\mathrm{P}\left(\delta_{n}^{\left(K_{1}\right)}=1, A_{n+1}^{\left(K_{1}\right)}=0\right)$.

Assumption 4.1 is less restrictive than [7, Assumptions 1(ii) and 2(i)] in the following sense: [7, Assumption 1(ii)] requires a time-invariant control rule for the controlled stream $\left\{B_{n}^{\left(K_{i}\right)}\right\}$, but Assumption 4.1(i) allows some time-variant control rules. Furthermore, [7, Assumption 1(ii)] requires $\mathrm{P}\left(B_{n+1}^{\left(K_{0}\right)}=k \mid X_{n}^{\left(K_{0}\right)}=K_{1}\right)=\mathrm{P}\left(B_{n+1}^{\left(K_{1}\right)}=k \mid X_{n}^{\left(K_{1}\right)}=\right.$ $K_{1}$ ) for all $k \in \mathbb{Z}_{+}$, but Assumption 4.1(i) requires only for $k=0$. Under [7, Assumption $2(\mathrm{i})]$, it is necessary that $\left\{\left(\delta_{n}^{\left(K_{i}\right)}, A_{n+1}^{\left(K_{i}\right)}\right)\right\}$ regenerates whenever the event $\left\{\delta_{n}^{\left(K_{i}\right)}=1, A_{n+1}^{\left(K_{i}\right)}=\right.$ $0\}$ occurs. But under Assumption 4.1(ii), it is sufficient that $\left\{\left(\delta_{n}^{\left(K_{i}\right)}, A_{n+1}^{\left(K_{i}\right)}\right)\right\}$ regenerates only when the event $\left\{\delta_{n}^{\left(K_{i}\right)}=1, A_{n+1}^{\left(K_{i}\right)}=0, X_{n}^{\left(K_{i}\right)}=K_{1}\right\}$ occurs. Furthermore, Assumption 4.1 does not require the stationarity of $\left\{\left(\delta_{n}^{\left(K_{i}\right)}, A_{n+1}^{\left(K_{i}\right)}, B_{n+1}^{\left(K_{i}\right)}\right)\right\}$, but [7, Assumptions 1(ii) and 2(i)] do.

The following corollary can be considered as a more general result than [7, Theorem 1] and it reduces to that if $\left\{X_{n}^{\left(K_{i}\right)}\right\}, i=0,1$, is stationary and ergodic and $\left\{\left(A_{n}^{\left(K_{i}\right)}, \delta_{n}^{\left(K_{i}\right)}\right)\right\}$ is stationary. 
Corollary 4.2. Suppose that Assumption 3.1 is satisfied with probability one. Further, suppose that $\mathscr{P}\left(\delta_{n}^{\left(K_{0}\right)}=1, A_{n+1}^{\left(K_{0}\right)}=0\right)>0$ with probability one. Then, under Assumption 4.1,

$$
\begin{gathered}
\mathscr{P}\left(X_{n}^{\left(K_{1}\right)}=j\right)=\frac{\mathscr{P}\left(\delta_{n}^{\left(K_{0}\right)}=1, A_{n+1}^{\left(K_{0}\right)}=0\right) \mathscr{P}\left(X_{n}^{\left(K_{0}\right)}=j\right)}{\mathscr{P}\left(X_{n}^{\left(K_{0}\right)} \leq K_{1}, \delta_{n}^{\left(K_{0}\right)}=1, A_{n+1}^{\left(K_{0}\right)}=0\right)}, \quad \text { for } j=0, \ldots, K_{1}-1, \\
\mathscr{P}\left(X_{n}^{\left(K_{1}\right)}=K_{1}\right)=1-\frac{\mathscr{P}\left(\delta_{n}^{\left(K_{0}\right)}=1, A_{n+1}^{\left(K_{0}\right)}=0\right) \mathscr{P}\left(X_{n}^{\left(K_{0}\right)} \leq K_{1}-1\right)}{\mathscr{P}\left(X_{n}^{\left(K_{0}\right)} \leq K_{1}, \delta_{n}^{\left(K_{0}\right)}=1, A_{n+1}^{\left(K_{0}\right)}=0\right)}
\end{gathered}
$$

hold with probability one, provided that the limits in (4.1) exist with probability one.

\section{Acknowledgment}

This research was supported by University IT Research Center Project.

\section{References}

[1] X. Chao and M. Miyazawa, On truncation properties of finite-buffer queues and queueing networks, Probability in the Engineering and Informational Sciences 14 (2000), no. 4, 409-423.

[2] P. Glasserman and W.-B. Gong, Time-changing and truncating $K$-capacity queues from one $K$ to another, Journal of Applied Probability 28 (1991), no. 3, 647-655.

[3] W.-B. Gong and K. Gong, On-line estimation of buffer size effect in $M / G / 1 / K$ queue and its application, Proceedings of 28th IEEE Conference on Decision and Control (Florida, 1989), vol. 2, IEEE, New York, 1989, pp. 1087-1092.

[4] F. Ishizaki, G. C. Lin, and T. Suda, An application of the proportional relation to concurrent estimation, Institute of Electrical and Electronics Engineers. Transactions on Automatic Control 48 (2003), no. 10, 1861-1865.

[5] F. Ishizaki and T. Takine, Loss probability in a finite discrete-time queue in terms of the steady state distribution of an infinite queue, Queueing Systems. Theory and Applications 31 (1999), no. 3-4, 317-326.

[6] J. Keilson and L. D. Servi, Blocking probability for M/G/1 vacation systems with occupancy level dependent schedules, Operations Research 37 (1989), no. 1, 134-140.

[7] N. Miyoshi and F. Ishizaki, A unified approach to the proportional relation for discrete-time singleserver queues, Queueing Systems. Theory and Applications 42 (2002), no. 2, 201-212.

Fumio Ishizaki: Department of Mathematics and Telecommunication Mathematics Research Center, Korea University, Seoul 136-701, Korea

E-mail address: fumio@ieee.org

Naoto Miyoshi: Department of Mathematical and Computing Sciences,

Tokyo Institute of Technology, Tokyo 152-8552, Japan

E-mail address: miyoshi@is.titech.ac.jp 


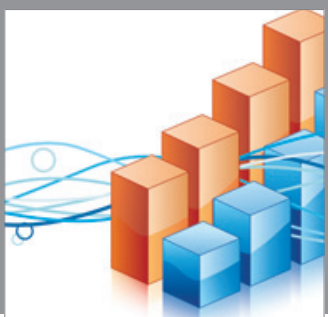

Advances in

Operations Research

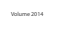

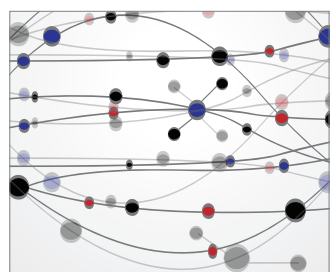

\section{The Scientific} World Journal
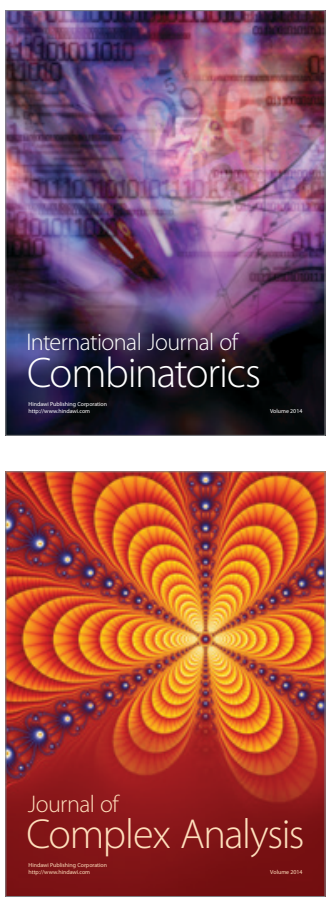

International Journal of

Mathematics and

Mathematical

Sciences
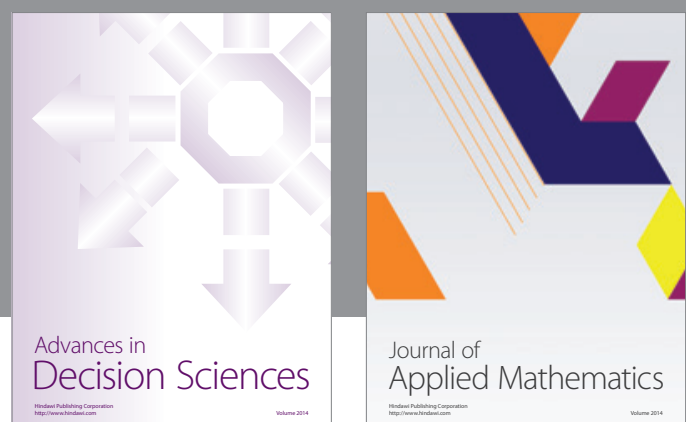

Journal of

Applied Mathematics
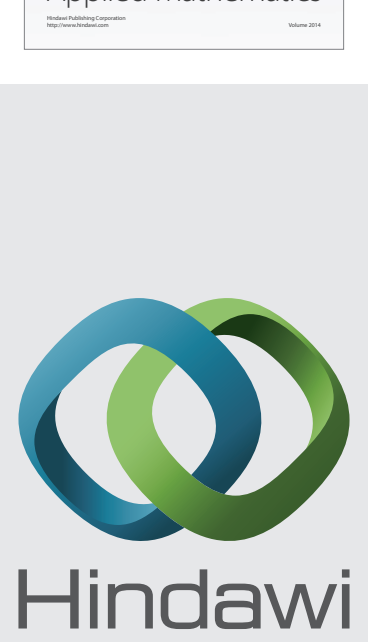

Submit your manuscripts at http://www.hindawi.com
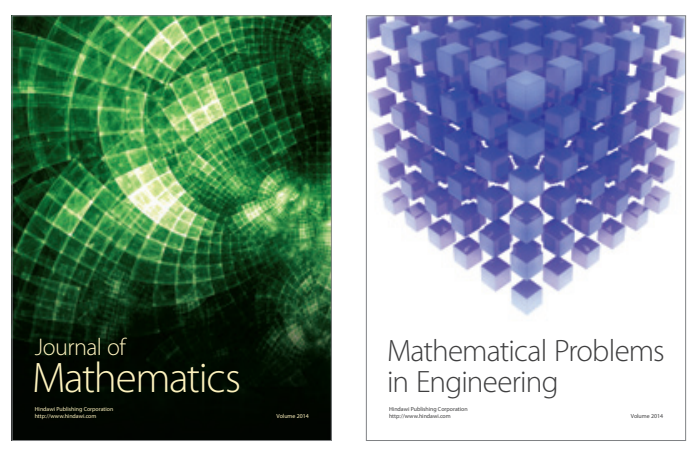

Mathematical Problems in Engineering
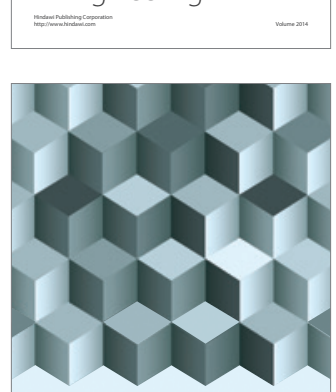

Journal of

Function Spaces
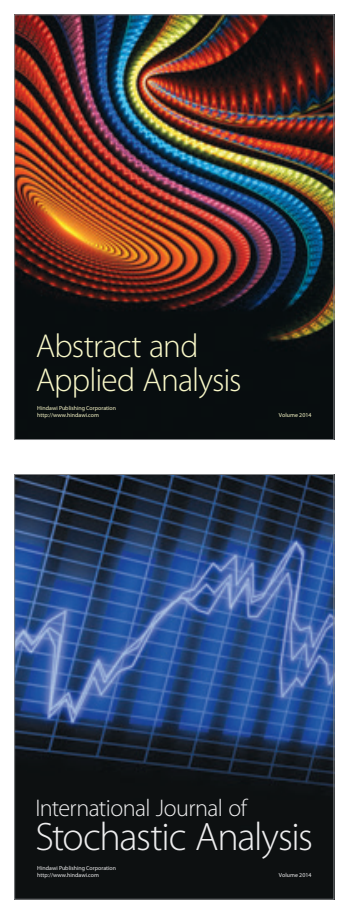

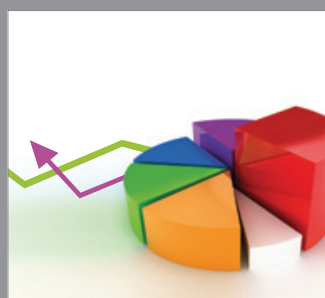

ournal of

Probability and Statistics

Promensencen
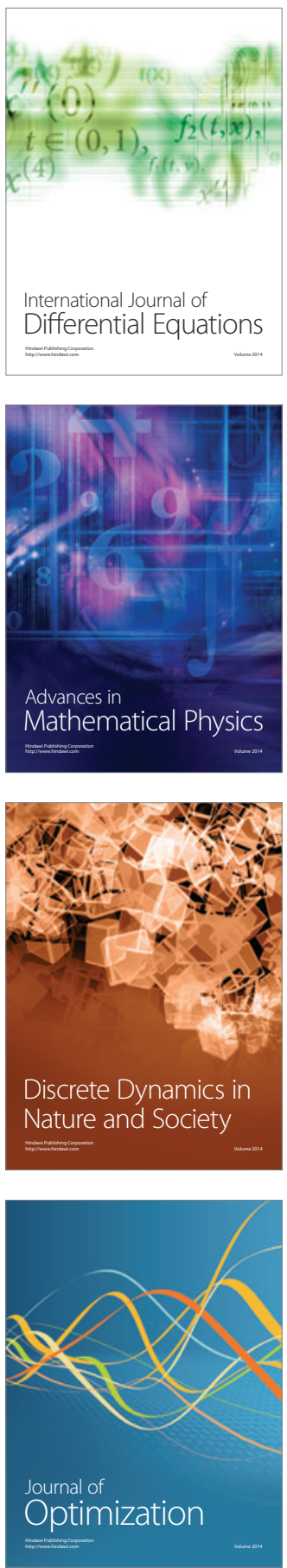